\title{
Stepwise Evolution of Photocatalytic Spinel- Structured (Co,Cr,Fe,Mn,Ni)304 High Entropy Oxides from First-Principles Calculations to Machine Learning
}

Chia-Chun Lin

National Cheng Kung University

Chia-Wei Chang

National Cheng Kung University

Chao-Cheng Kaun

Academia Sinica

Yen-Hsun Su ( $\square$ yhsu@mail.ncku.edu.tw)

National Cheng Kung University

\section{Research Article}

Keywords: Spinel structure, High entropy oxides, First-principles calculations, Machine learning

Posted Date: June 1st, 2021

DOI: https://doi.org/10.21203/rs.3.rs-565930/v1

License: (c) (1) This work is licensed under a Creative Commons Attribution 4.0 International License.

Read Full License 
Stepwise Evolution of Photocatalytic Spinel-Structured (Co,Cr,Fe,Mn,Ni) ${ }_{3} \mathrm{O}_{4}$ High Entropy Oxides from First-Principles Calculations to Machine Learning

Chia-Chun Lin ${ }^{1,2}$, Chia-Wei Chang ${ }^{1}$, Chao-Cheng Kaun ${ }^{2 *}$, Yen-Hsun Su${ }^{*}$

${ }^{1}$ Department of Materials Science and Engineering, National Cheng Kung University, Tainan 70101, Taiwan

${ }^{2}$ Research Center for Applied Sciences, Academia Sinica, Taipei 11529, Taiwan

${ }^{*}$ Corresponding author’s e-mail: yhsu@mail.ncku.edu.tw, kauncc@gate.sinica.edu.tw 


\section{$\underline{\text { Abstract }}$}

High entropy oxides (HEOx) are novel materials, which increase the potential application in the fields of energy and catalysis. However, a series of HEOx is too novel to evaluate the synthesis properties, including formation and fundamental properties. Combining first-principles calculations with machine learning (ML) techniques, we predict the lattice constants and formation energies of spinel-structured photocatalytic $\mathrm{HEOx},(\mathrm{Co}, \mathrm{Cr}, \mathrm{Fe}, \mathrm{Mn}, \mathrm{Ni})_{3} \mathrm{O}_{4}$, for stoichiometric and nonstoichiometric structures. The effects of site occupation by different metal cations in the spinel structure are obtained through first-principles calculations and ML predictions. Our predicted results show that the lattice constants of these spinel-structured oxides are composition-dependent and that the formation energies of those oxides containing $\mathrm{Cr}$ atoms are low. The computing time and computing energy can be greatly economized through the tandem approach of first-principles calculations and ML.

\section{Keywords}

Spinel structure, High entropy oxides, First-principles calculations, Machine learning 


\section{$\underline{\text { Introduction }}$}

Recently, high entropy materials (HEMs) have been gradually getting more and more attention due to their novel and particular properties. Functional materials are found a brand new direction for developing via the discovery of HEMs. ${ }^{1}$ HEOx are intensively and widely investigated in the fields of energy storage or catalyst for the environment, such as lithium-ion batteries ${ }^{2-5}$, oxygen evolution reactions $(\mathrm{OERs})^{6,7}$, and catalyst activity ${ }^{8-11}$

The first HEOx, a (Co,Cu,Mg,Ni,Zn)O equimolar single-phase (Fm $\overline{3 m})$ rock-salt structure, was synthesized by Rost et al. ${ }^{12}$ in 2015 and further studied by Bérardan et al. ${ }^{13}$ The spinel-type structure HEOx is firstly synthesized and researched ${ }^{14}$. The physical properties of $(\mathrm{Co}, \mathrm{Cu}, \mathrm{Mg}, \mathrm{Ni}, \mathrm{Zn}) \mathrm{O}$ powders, such as the dielectric constants ${ }^{15}$ and magnetic properties ${ }^{16}$ were also addressed. These HEOx compounds are attractive because they have high ionic conductivity in an alkali-doped compound, which are utilized in Li-ion ${ }^{17,18}$ and Li-S batteries ${ }^{19}$ for energy storage. In addition to the rock-salt HEOx, other crystal structures, such as perovskite ${ }^{20}$ fluorite $^{21,22}$, and spinel ${ }^{14,23,24}$ HEOx have been successfully synthesized and developed recently. The typical spinels ${ }^{25}$ are described as $\mathrm{AB}_{2} \mathrm{O}_{4}$, where metal cations at A sites occupy the center of the tetrahedral position, metal cations at B sites occupy the center of the octahedral position, and anions locate at the vertexes of the polyhedron. Spinelstructured HEOx were firstly synthesized and studied for many fantastic properties. ${ }^{14,23,24,26-29}$ A singlephase $(\mathrm{Fd} \overline{3 m})$ spinel-structured HEOx, $\left(\mathrm{Co}, \mathrm{Cr}, \mathrm{Fe}, \mathrm{Mn}, \mathrm{Ni}_{3} \mathrm{O}_{4}\right.$, was synthesized ${ }^{14}$ in 2018 . The defect structures and chemical diffusion in spinel-structured $(\mathrm{Co}, \mathrm{Cr}, \mathrm{Fe}, \mathrm{Mn}, \mathrm{Ni})_{3} \mathrm{O}_{4}$ were further proposed ${ }^{24}$, the findings of which indicated the complexity of the defect structure in these high-entropy spinels.

The defect structure and chemical diffusion in HEMs can be addressed by using first-principles calculations. For example, the formation and migration of intrinsic defects in $\mathrm{CrCoFeNi}$ high-entropy alloys (HEAs) were studied. ${ }^{30}$ Furthermore, the formation energy was used to determine how favorable the structure is to form a particular phase as compared with the pure stable phases. The relative crystal stability of HEMs can be determined by the formation energy. 
The physical properties of crystal structures, such as lattice constants and formation energy, can be calculated and determined from first principles. Various crystal structures have been discussed and demonstrated. ${ }^{31-34}$ In the case of HEMs, there are more elemental species, and the occupation situations of multi-element in lattice positions become numerous. Hence, the calculation demand for HEMs is heavy and complicated. The interest and curiosity in calculating stoichiometric compounds (i.e., compounds with an integer ratio of the number of atoms) and non-stoichiometric compounds $\mathrm{s}^{35-37}$ have been provoked along with the development of HEMs. Both equimolar and non-equimolar HEOx are considered to be worthy of exploration.

The interactions in HEOx are very complicated due to the random distribution of ions with different electronic configurations. Therefore, the calculation loading to determine the formation energy and lattice constant for HEOx is heavy. The first-principles calculations of the spinel-structured HEOx are thus still limited so far. However, ML has been used to study HEMs because it can be used to quickly and efficiently infer the properties of materials, such as hardness ${ }^{38}$ and phase selection ${ }^{39}$. These studies show that ML describes the correlation between material characteristics. ML can accurately predict the properties of HEMs.

Density functional theory $(\mathrm{DFT})^{40,41}$-based first principles calculations are widely used in physics, chemistry, and material sciences, for which the concept involved is a quantum mechanical modeling method. Their computational loads are heavy whether computing time or computing power are considered. However, the DFT can be combined with ML to reduce such loads, where ML utilizes computer algorithms to automatically predict the physics, chemistry, and material properties through DFT experiences.

Here, we use DFT-based first-principles calculations to calculate the spinel-structured multielement compounds to determine their lattice constants and formation energies with various element species and different occupying positions. Furthermore, in the case of the non-stoichiometric HEOx compounds, ML is used to build mathematical models based on the training data to avoid the huge computational load caused by their superlattice structures. Two arithmetic methods of ML, back- 
propagation network (BPN) and genetic algorithm neural network (GANN), are used and compared. We also compare the computing time and the accuracy of two different approaches, DFT calculations and ML. The combination of the first principles and ML approaches used to explore a nonstoichiometric compound computation model is demonstrated to predict the existence of HEOx crystal structures. Fig. 1 shows the schematic of the main concept.

\section{$\underline{\text { Results and discussion }}$}

\section{Decision flow}

Supplementary Fig. 1 shows the workflow of the HEOx investigation. The model building method and ML simulation of machine learning models are discussed. The results of BPN and GANN models are compared to evaluate the way we build the models. First, we set the metal elements in the A and B sites of the spinel structure as the independent variables. The lattice constants and formation energies obtained from DFT results shown in Supplementary Table 1 are set as the dependent variables. The above data are then used as the ML input training data. In order to choose a useful machine learning model, we use low data test to compare the widely used BPN with GANN models for complicated cases. Root mean square error (RMSE) values and scatter plots are used to determine and choose a suitable model. The prediction of the new spinel structure is then performed using the lower RMSE model. Finally, the ML results of the new spinel-structured HEOx are compared with the DFT results.

\section{ML-model selection and performance}

To construct machine-learning potential for this research, we first generate a DFT dataset to fit the physical properties of the spinel-structured HEOx. The initial training low data set includes 18 currently reported configurations of the major spinels ${ }^{42}$, as shown in Supplementary Table 2. These configurations are in the ground states obtained from the DFT calculations (see the "Methods" section for details). Supplementary Fig. $2 \mathrm{a}$ and $\mathrm{b}$ show the lattice constants and formation energies of those major spinels in the low data test, respectively. The metal elements $\mathrm{Co}, \mathrm{Cr}, \mathrm{Fe}, \mathrm{Mn}$, and $\mathrm{Ni}$ in the A and $\mathrm{B}$ sites of the spinel structure are represented in the $\mathrm{X}$-axis and Y-axis, respectively. The colors 
represented the lattice constants and formation energies obtain from ML. Supplementary Fig. 2a shows that the value of lattice constants a, b, and c of these spinel oxides (in Supplementary Table 2) can be different. Supplementary Fig. $2 \mathrm{~b}$ shows that the formation energies of all spinel oxides are negative, suggesting that they are in stable states. Cr included structures have lower formation energies, about $1800 \mathrm{~kJ} / \mathrm{mole}$, as compared to the other compounds.

Supplementary Fig. 3a and $\mathrm{b}$ show the RMSE values of the lattice constants and formation energies, respectively. The RMSE values $=\sqrt{\frac{1}{n} \sum_{i=1}^{n}\left(y_{i}-\hat{y}_{i}\right)^{2}}$, where $\mathrm{y}_{\mathrm{i}}$ and $\hat{\mathrm{y}}_{\mathrm{i}}$ are the real and predicted values, respectively. The number of learning cycles is set to 1 million to ensure that the RMSE has a good convergence. The RMSE values of the lattice constants obtained from the BPN and GANN models decrease as the training progresses and finally converge to 0.0394 and 0.0071 , respectively. The RMSE values of the formation energies obtained from the BPN and GANN models also decrease as the training progresses and converge to 0.0831 and 0.0182 , respectively. The latter model, GANN, thus is better than BPN model. The scatter plots of the lattice constants, a, b, and c obtained from the BPN and GANN models are shown in Supplementary Fig. 3c and d, respectively. Supplementary Fig. 3e and $f$ are the scatter plots of the formation energies obtained from the BPN and GANN models, respectively. The data from the latter model is closer to the regression line than that of the former one, which means GANN model has higher accuracy in this case. Therefore, we use the GANN model as the ML method in the following studies.

\section{Prediction of the new spinel structure}

The predicted lattice constants and formation energies of the spinel-structured HEOx and the new spinel structures are plotted in Figs. 2 to 5. In order to study the influence of the composition of HEOx on lattice constants and formation energies in more detail, we divide $(\mathrm{Co}, \mathrm{Cr}, \mathrm{Fe}, \mathrm{Mn}, \mathrm{Ni}){ }_{3} \mathrm{O}_{4}$ into two categories according to different metal cations in the A and B sites (Figs. 2 and 4), and the different proportion of metal cations in the A and B sites (Figs. 3 and 5) in the same composition. Fig. 2 shows the predicted lattice constants, where the metal elements in the A and B sites of the spinel structure can 
be a single metal element or multiple metal elements. The colors represented the lattice constants $a, b$, and $\mathrm{c}$ are predicted from the GANN model. Lattice constants $\mathrm{a}, \mathrm{b}$, and $\mathrm{c}$ have different but similar values.

Because the spinel structure consists of various proportions of the elements, the proportion of one metal element in the A (B) site of the spinel structure is set as $\mathrm{x}(\mathrm{y})$, where $\mathrm{x}(\mathrm{y})$ increases from 0 to 1 by an interval of 0.1 . The total proportion of the other metal elements in $\left(\mathrm{Co}, \mathrm{Cr}, \mathrm{Fe}, \mathrm{Mn}, \mathrm{Ni}_{3} \mathrm{O}_{4}\right.$ is set as 1-x (y), as shown in Supplementary Table 3. The colors shown in Fig. 3 represented the lattice constants $\mathrm{a}, \mathrm{b}$, and $\mathrm{c}$ are predicted from the GANN model. The results show that the lattice constants $\mathrm{a}, \mathrm{b}$, and c have different but similar values.

The predicted formation energies of the spinel-structured HEOx or new spinel structure oxides are presented in Figs. 4 and 5. The colors in Fig. 4 indicate that the formation energies predicted by the GANN model are negative. Compared with other structures, the formation energies for the Crincluded structures are lower, which is consistent with the results obtained in the low data test. The colors shown in Fig. 5 are corresponding to the formation energies predicted by the GANN model, which are determined by the proportions of different metal elements in both A and B sites. However, the B-site-dependence of the formation energy is stronger than the A-site- dependence. The smaller the $\mathrm{y}$ value of the metal elements in B sites becomes, the lower the formation energy of the spinel structure is. In contrast, the formation energy of the spinel structures with $\mathrm{Cr}$ in the $\mathrm{B}$ sites follows the opposite tendency.

\section{Comparison of the calculated and predicted results}

Verifying the accuracy of the predicted results is also critical for the ML. To compare and verify the calculated and predicted results from the ML model, we design some spinel-structured HEOx with different composition shown in Supplementary Table 1. The structures are composed of metal elements in all of the various situations, as shown in Supplementary Table 4. The lattice constants and formation energies of the new spinel structures obtained via the DFT calculations and ML methods are shown in Figs. 6a and b, respectively. Fig. 6a shows that the lattice constants predicted by the GANN model 
match well with the calculations. The average lattice parameters of the label from I to IV are predicted respectively to be $8.324,8.294,8.302$, and $8.317 \AA$ (the variation are calculated to be $\pm 0.024, \pm 0.032$, \pm 0.007 , and $\pm 0.009 \AA$ ) which are within $2 \%$ deviation for the DFT calculations. As shown in Fig. $6 \mathrm{~b}$, each supercell has different predicted formation energy: Labels I to IV are -849.81, -763.00, -921.86, and $-978.91 \mathrm{~kJ} / \mathrm{mole}$, respectively. The formation energy of labels I to IV calculated by using DFT are $-851.37,-762.93,-921.70$, and $-977.02 \mathrm{~kJ} /$ mole, respectively. The predicted results are within $1 \%$ deviation for the DFT results. Thus, both the lattice constants and the formation energy of the new spinel structure HEOx from the ML methods concur well with the results from the DFT calculations. The results of DFT calculations and ML methods of big-size supercell of spinel-structured HEOx are also shown in Supplementary Table 1.

The spinel-typed structure HEOx was firstly synthesized and researched for fantasy properties in recent years. If the compounds have a crystal structure following the stoichiometric chemical formula, the structure and electronic structure can be simulated through the calculation based on the first principles, and the properties can be determined. When the composition comes to a nonstoichiometric chemical formula, a big-size supercell is necessary for the DFT calculation, and thus a more complicated approach is required to reduce computing time and save electric power energy. The unit cell of the materials is thus replicated until the composition integrates into a stoichiometric superlattice. After the non-stoichiometric compound converts into a huge crystal structure, a stoichiometric superlattice, the calculation can progress to the first-principles calculations. That is to say, the calculation of the non-stoichiometric compound in DFT is necessary through a setup of a heavy-loading supercell structure, which is limited by computing performance and results in energy consumption. A comparison of the computing results of the spinel structures using DFT and ML are shown in Supplementary Table 1.

The spinel structure, $\mathrm{AB}_{2} \mathrm{O}_{4}$, including cubic, tetragonal and orthorhombic structures are taken into consideration, and they have 56, 28, and 28 atoms in a unit cell, respectively. The cubic, tetragonal and orthorhombic structures of stoichiometry/non-stoichiometry $\mathrm{AB}_{2} \mathrm{O}_{4}$ are also considered by 
installing a single cell and a supercell. The computing time required for DFT calculations differs from the degrees of difficulty in the self-consistent calculation of the electronic density matrix. The lattice constants and formation energies of stoichiometry/non-stoichiometry $\mathrm{AB}_{2} \mathrm{O}_{4}$ predicted using $\mathrm{ML}$ are consistent with those calculated using DFT directly. ML also has the technological advance to deal with the crystallographic in a non-stoichiometric form. Thus, computing time and power can be saved and the process is more efficient.

Since the fabrication and character simulation of HEOx is in a state of urgent, the prediction of the possible HEOx crystal structure is very important and the priority. The calculation and modeling of spinel-typed crystal structures are carried out, demonstrated, compared, and discussed by using the combination of first principles and the ML method in this research. This can potentially greatly accelerate the development of functional HEMs.

\section{Conclusions}

We propose an efficient ML method by which to obtain the lattice constants and formation energies of photocatalytic spinel-structured HEOx and multi-element oxides. We assign $\mathrm{Cr}, \mathrm{Co}, \mathrm{Fe}$, $\mathrm{Mn}$, and $\mathrm{Ni}$ in the lattice sites and capture the electronic configuration well by using a random population of supercells. After the DFT calculations, the lattice constants of the spinels are given, and the formation energies of the equilibrium lattice structures are negative, which are chosen as training data for ML modeling. Our results show that the occupation of different metal cations in the A and B sites in the spinel-structured HEOx influence the lattice constants and formation energies. Furthermore, the formation energy of the structure is inversely proportional to the amount of $\mathrm{Cr}$, and the $\mathrm{ML}$ predictions are consistent with the DFT calculations (within a $2 \%$ and $1 \%$ deviation for the lattice constants and formation energies, respectively), which suggests that the process and design used in this study are feasible. This work is provided with a new alternative way to obtain the physical properties of spinel-structured HEOx and can also be extended to other structured HEOx materials to 
predict their properties efficiently following a combination of DFT calculations and model building using the ML arithmetic method.

Furthermore, we provide a database of calculated lattice constants and formation energies for spinel structures. This database can be used to understand the properties of spinel-structured highentropy materials and other spinel-structured oxides. The database are provided with opportunities for data-intensive materials science and the calculation of the properties of a near-infinite variety of spinelstructured materials with $\mathrm{Co}, \mathrm{Cr}, \mathrm{Fe}, \mathrm{Mn}$, and Ni metals. The application of ML techniques led to the identification of structural features that are the key to the spinels. This work thus not only enhance the theoretical understanding of spinel-structures oxides but also accelerate the discovery of stable spinelstructured HEOx or other spinel-structured oxides.

\section{$\underline{\text { Methods }}$}

\section{First-principles calculations}

These calculations, based on the DFT, were performed by using the Vienna Ab-initio simulation software package (VASP) $)^{43,44}$, with the projector augmented wave (PAW) ${ }^{44,45}$ pseudopotential and the generalized gradient approximation (GGA) used to determine the exchange-correlation energy in the form of Perdew-Becke-Ernzehof ${ }^{46,47}$. Spin-polarization effects were also considered.

Calculations were carried out by using face-centered cubic (FCC) supercells containing 56 atoms, body-centered tetragonal (BCT) supercells containing 28 atoms, and face-centered orthorhombic (FCO) supercells containing 28 atoms. We expected that using simple scripts describing $\mathrm{Co}, \mathrm{Co}, \mathrm{Fe}, \mathrm{Mn}$, and $\mathrm{Ni}$ were randomly assigned to the lattice sites to produce similar results to the special quasi-random structure $(\mathrm{SQS})^{48}$ method. The initial lattice parameters for FCC structures $(\mathrm{Fd} \overline{3 \mathrm{~m}})$ with $8.150 \AA$ and BCT structures (I4 $1 / \mathrm{amd})$ with $\mathrm{a}=5.903 \AA$ and $\mathrm{c}=8.348 \AA$ and $\mathrm{FCO}$ structures (Imma) with $\mathrm{a}=6.002 \AA, \mathrm{b}=6.017 \AA$, and $\mathrm{c}=8.301 \AA$ were set. A $520 \mathrm{eV}$ plane wave cutoff and the $3 \times$ $3 \times 3,4 \times 4 \times 3$, and $4 \times 4 \times 3$ Monkhorst-Pack k-point grid were used for the calculations. Structure cards for VASP were shown in the Supplementary Information. 
All structures were relaxed. The total energies and lattice constants were analyzed. The formation energy was used to determine a particular phase compared with the pure stable phases. The ground state formation energy is estimated using the following equation:

$$
E^{\text {form }}=E^{A B_{2} O_{4}}-\sum c_{i} E_{i}
$$

where $E^{A B_{2} O_{4}}$ is the total energies of spinel structure $\left(\mathrm{AB}_{2} \mathrm{O}_{4}\right), E_{i}$ is the total energies of each element $i$ in its stable phase, and $C_{i}$ is the concentration of each element.

\section{Machine learning (ML) algorithms}

The Super PCNeuron 5.0 (SPCN 5.0) package $^{49}$ was used. The BPN model consists of three steps: Create the input file for training, learning and outputing data. First, the metal elements of $(\mathrm{Co}, \mathrm{Cr}, \mathrm{Fe}, \mathrm{Mn}, \mathrm{Ni})_{3} \mathrm{O}_{4}$ in $\mathrm{A}$ or $\mathrm{B}$ sites were set as the independent variables, $\mathrm{X}$, and the lattice constants and formation energy of the spinels from DFT calculations were set as the dependent variables, Y, as an input file. Next, the hidden layers were used to capture the characteristics of the training data and reflect on the relevant parameters. The number of hidden layers was set to 2 , and the number of neurons in each layer was set to 20 based on the decreasing mean square error in the trial and error process. Lastly, the output layer was used to represent the output variables of the network. High training and testing accuracy was achieved by modifying the training and parameters.

The GANN combines the characteristics of artificial neural networks (ANN) with genetic algorithm (GA). The input parameters included the type and proportion of metal elements in the A or B sites of the spinel structure as well as the DFT calculations of the lattice constants and formation energies. The number of learning cycles was set to 1 million based on the decreasing mean square err in the trial-and-error process. The three main operators of GANN are reproduction, crossover, and mutation. The crossover probability and mutation probability were 0.75 and 0.01 , respectively. Furthermore, 3 hidden layers were used, and the number of neurons in each layer was set to 20, 15, and 25 , respectively.

\section{$\underline{\text { Data Availability }}$}


The reported major spinels can be obtained and downloaded on the Materials Project ${ }^{50}$ website (www.materialsproject.org). In addition, the Materials Project website also provides information on the simulation parameters, crystal structure, electronic properties, and other properties of these materials. VASP version 5.4.4 used to perform the DFT calculations is proprietary code. The SPCN 5.0 package used to carry out all algorithms is proprietary software available at http://www.ce.tku.edu.tw/ icyeh/spcn/.

\section{$\underline{\text { Author contributions }}$}

All authors contributed to the outline of the workflow. Chia-Chun Lin performed the density functional theory calculations and machine learning training and predictions. All authors performed the literature search to identify known spinel structures consisting of $\mathrm{Co}, \mathrm{Cr}, \mathrm{Fe}, \mathrm{Mn}$, and Ni metals in the dataset. Chao-Cheng Kaun and Yen-Hsun Su were involved in supervising and planning the work. All authors contributed to the writing of the manuscript.

\section{Conflict of Interest}

The authors declare no conflict of interest.

\section{$\underline{\text { Acknowledgments }}$}

This work was supported in part by the Ministry of Science and Technology, Taiwan, under Grants MOST-109-2221-E-006-024-MY3/109-2224-E-006-009/109-2224-E-006-007/107-2112-M001-036-MY3, Academia Sinica, Taiwan under Grant AS-iMATE-109-41, and we wish to thank the National Center for High-performance Computing for access to their computing time and facilities.

\section{References}

1 Dragoe, N. \& Bérardan, D. Order emerging from disorder. Science 366, 573, doi:10.1126/science.aaz1598 (2019). 

3400, doi:10.1038/s41467-018-05774-5 (2018).

3 Qiu, N. et al. A high entropy oxide (Mg0.2Co0.2Ni0.2Cu0.2Zn0.2O) with superior lithium storage performance. Journal of Alloys and Compounds 777, 767-774, doi:https://doi.org/10.1016/i.jallcom.2018.11.049 (2019).

4 Wang, Q. et al. High entropy oxides as anode material for Li-ion battery applications: A practical approach. Electrochemistry Communications 100, 121-125, doi:https://doi.org/10.1016/j.elecom.2019.02.001 (2019). Wang, Q. et al. Multi-anionic and -cationic compounds: new high entropy materials for advanced Li-ion batteries. Energy \& Environmental Science 12, 2433-2442, doi:10.1039/C9EE00368A (2019).

Dai, W., Lu, T. \& Pan, Y. Novel and promising electrocatalyst for oxygen evolution reaction based on MnFeCoNi high entropy alloy. Journal of Power Sources 430, 104-111, doi:https://doi.org/10.1016/i.jpowsour.2019.05.030 (2019). Huang, K. et al. Exploring the impact of atomic lattice deformation on oxygen evolution reactions based on a sub-5 $\mathrm{nm}$ pure face-centred cubic high-entropy alloy electrocatalyst. Journal of Materials Chemistry A 8, 11938-11947, doi:10.1039/D0TA02125C (2020).

8 Chen, H. et al. Entropy-stabilized metal oxide solid solutions as $\mathrm{CO}$ oxidation catalysts with high-temperature stability. Journal of Materials Chemistry A 6, 11129-11133, doi:10.1039/C8TA01772G (2018).

9 Chen, H. et al. Mechanochemical Synthesis of High Entropy Oxide Materials under Ambient Conditions: Dispersion of Catalysts via Entropy Maximization. ACS Materials Letters 1, 83-88, doi:10.1021/acsmaterialslett.9b00064 (2019).

10 Zhang, Z. et al. Mechanochemical Nonhydrolytic Sol-Gel-Strategy for the Production of Mesoporous Multimetallic Oxides. Chemistry of Materials 31, 5529-5536, doi:10.1021/acs.chemmater.9b01244 (2019).

11 Okejiri, F. et al. Room-Temperature Synthesis of High-Entropy Perovskite Oxide Nanoparticle Catalysts through Ultrasonication-Based Method. ChemSusChem 13, 111-115, doi:https://doi.org/10.1002/cssc.201902705 (2020).

12 Rost, C. M. et al. Entropy-stabilized oxides. Nature Communications 6, 8485, doi:10.1038/ncomms9485 (2015).

13 Berardan, D., Meena, A. K., Franger, S., Herrero, C. \& Dragoe, N. Controlled Jahn-Teller distortion in (MgCoNiCuZn)O-based high entropy oxides. Journal of Alloys and Compounds 704, 693-700, doi:https://doi.org/10.1016/i.jallcom.2017.02.070 (2017).

Dąbrowa, J. et al. Synthesis and microstructure of the (Co,Cr,Fe, Mn, Ni)3O4 high entropy oxide characterized by spinel structure. Materials Letters 216, 32-36, doi:https://doi.org/10.1016/i.matlet.2017.12.148 (2018). Bérardan, D., Franger, S., Dragoe, D., Meena, A. K. \& Dragoe, N. Colossal dielectric constant 
in high entropy oxides. physica status solidi (RRL) - Rapid Research Letters 10, 328-333, doi:https://doi.org/10.1002/pssr.201600043 (2016).

Jimenez-Segura, M. P. et al. Long-range magnetic ordering in rocksalt-type high-entropy oxides. Applied Physics Letters 114, 122401, doi:10.1063/1.5091787 (2019).

17 Bérardan, D., Franger, S., Meena, A. K. \& Dragoe, N. Room temperature lithium superionic conductivity in high entropy oxides. Journal of Materials Chemistry A 4, 9536-9541, doi:10.1039/C6TA03249D (2016).

18 Osenciat, N. et al. Charge compensation mechanisms in Li-substituted high-entropy oxides and influence on Li superionic conductivity. Journal of the American Ceramic Society 102, 6156-6162, doi:https://doi.org/10.1111/jace.16511 (2019).

19 Zheng, Y. et al. A high-entropy metal oxide as chemical anchor of polysulfide for lithiumsulfur batteries. Energy Storage Materials 23, 678-683, doi:https://doi.org/10.1016/i.ensm.2019.02.030 (2019).

20 Sarkar, A. et al. Rare earth and transition metal based entropy stabilised perovskite type oxides. Journal of the European Ceramic Society 38, 2318-2327, doi:https://doi.org/10.1016/i.jeurceramsoc.2017.12.058 (2018).

21 Chen, K. et al. A five-component entropy-stabilized fluorite oxide. Journal of the European Ceramic Society 38, 4161-4164, doi:https://doi.org/10.1016/i.jeurceramsoc.2018.04.063 (2018).

22 Gild, J. et al. High-entropy fluorite oxides. Journal of the European Ceramic Society 38, 35783584, doi:https://doi.org/10.1016/i.jeurceramsoc.2018.04.010 (2018).

23 Mao, A. et al. Facile synthesis and ferrimagnetic property of spinel (CoCrFeMnNi)3O4 highentropy oxide nanocrystalline powder. Journal of Molecular Structure 1194, 11-18, doi:https://doi.org/10.1016/i.molstruc.2019.05.073 (2019).

24 Grzesik, Z. et al. Defect structure and transport properties of ( $\mathrm{Co}, \mathrm{Cr}, \mathrm{Fe}, \mathrm{Mn}, \mathrm{Ni}) 3 \mathrm{O} 4$ spinelstructured high entropy oxide. Journal of the European Ceramic Society 40, 835-839, doi:https://doi.org/10.1016/i.jeurceramsoc.2019.10.026 (2020).

25 Bragg, W. H. The Structure of Magnetite and the Spinels. Nature 95, 561-561, doi:10.1038/095561a0 (1915).

26 Musicó, B. et al. Tunable magnetic ordering through cation selection in entropic spinel oxides. Physical Review Materials 3, 104416, doi:10.1103/PhysRevMaterials.3.104416 (2019).

27 Mao, A. et al. A new class of spinel high-entropy oxides with controllable magnetic properties. Journal of Magnetism and Magnetic Materials 497, 165884, doi:https://doi.org/10.1016/i.jmmm.2019.165884 (2020).

28 Mao, A. et al. A novel six-component spinel-structure high-entropy oxide with ferrimagnetic property. Journal of Magnetism and Magnetic Materials 503, 166594, doi:https://doi.org/10.1016/i.jmmm.2020.166594 (2020). 
Wang, D. et al. Spinel-structured high entropy oxide (FeCoNiCrMn)3O4 as anode towards superior lithium storage performance. Journal of Alloys and Compounds 844, 156158, doi:https://doi.org/10.1016/i.jallcom.2020.156158 (2020). Middleburgh, S. C., King, D. M., Lumpkin, G. R., Cortie, M. \& Edwards, L. Segregation and migration of species in the CrCoFeNi high entropy alloy. Journal of Alloys and Compounds 599, 179-182, doi:https://doi.org/10.1016/j.jallcom.2014.01.135 (2014). Jasiewicz, K., Cieslak, J., Kaprzyk, S. \& Tobola, J. Relative crystal stability of AlxFeNiCrCo high entropy alloys from XRD analysis and formation energy calculation. Journal of Alloys and Compounds 648, 307-312, doi:https://doi.org/10.1016/i.jallcom.2015.06.260 (2015). Jiang, L. Q. et al. Prediction of lattice constant in cubic perovskites. Journal of Physics and Chemistry of Solids 67, 1531-1536, doi:https://doi.org/10.1016/i.jpcs.2006.02.004 (2006). Emery, A. A. \& Wolverton, C. High-throughput DFT calculations of formation energy, stability and oxygen vacancy formation energy of ABO3 perovskites. Scientific Data 4, 170153, doi:10.1038/sdata.2017.153 (2017).

Takahashi, K., Takahashi, L., Baran, J. D. \& Tanaka, Y. Descriptors for predicting the lattice constant of body centered cubic crystal. The Journal of Chemical Physics 146, 204104, doi:10.1063/1.4984047 (2017). Dubey, P. \& Kaurav, N. (2019). Lo, K.-C., Chang, Y.-J., Murakami, H., Yeh, J.-W. \& Yeh, A.-C. An oxidation resistant refractory high entropy alloy protected by CrTaO4-based oxide. Scientific Reports 9, 7266, doi:10.1038/s41598-019-43819-x (2019).

37 Ye, B., Wen, T., Huang, K., Wang, C.-Z. \& Chu, Y. First-principles study, fabrication, and characterization of ( $\mathrm{HfO} .2 \mathrm{ZrO} .2 \mathrm{Ta} 0.2 \mathrm{Nb0}$.2Ti0.2) C high-entropy ceramic. Journal of the American Ceramic Society 102, 4344-4352, doi:https://doi.org/10.1111/jace.16295 (2019). Wen, C. et al. Machine learning assisted design of high entropy alloys with desired property. Acta Materialia 170, 109-117, doi:https://doi.org/10.1016/i.actamat.2019.03.010 (2019). Huang, W., Martin, P. \& Zhuang, H. L. Machine-learning phase prediction of high-entropy alloys. Acta Materialia 169, 225-236, doi:https://doi.org/10.1016/i.actamat.2019.03.012 (2019).

40 Hohenberg, P. \& Kohn, W. Inhomogeneous Electron Gas. Physical Review 136, B864-B871, doi:10.1103/PhysRev.136.B864 (1964).

41 Kohn, W. \& Sham, L. J. Self-Consistent Equations Including Exchange and Correlation Effects. Physical Review 140, A1133-A1138, doi:10.1103/PhysRev.140.A1133 (1965).

42 Zhao, Q., Yan, Z., Chen, C. \& Chen, J. Spinels: Controlled Preparation, Oxygen Reduction/Evolution Reaction Application, and Beyond. Chemical Reviews 117, 1012110211, doi:10.1021/acs.chemrev.7b00051 (2017).

43 Kresse, G. \& Furthmüller, J. Efficient iterative schemes for ab initio total-energy calculations using a plane-wave basis set. Physical Review B 54, 11169-11186, 
doi:10.1103/PhysRevB.54.11169 (1996).

44 Kresse, G. \& Furthmüller, J. Efficiency of ab-initio total energy calculations for metals and semiconductors using a plane-wave basis set. Computational Materials Science 6, 15-50, doi:https://doi.org/10.1016/0927-0256(96)00008-0 (1996).

45 Blöchl, P. E. Projector augmented-wave method. Phys Rev B Condens Matter 50, 1795317979, doi:10.1103/physrevb.50.17953 (1994).

46 Perdew, J. P. et al. Atoms, molecules, solids, and surfaces: Applications of the generalized gradient approximation for exchange and correlation. Physical Review B 46, 6671-6687, doi:10.1103/PhysRevB.46.6671 (1992).

47 Perdew, J. P., Burke, K. \& Ernzerhof, M. Generalized Gradient Approximation Made Simple. Phys Rev Lett 77, 3865-3868, doi:10.1103/PhysRevLett.77.3865 (1996).

48 Gao, M. C., Niu, C., Jiang, C. \& Irving, D. L. in High-Entropy Alloys: Fundamentals and Applications （eds Michael C. Gao, Jien-Wei Yeh, Peter K. Liaw, \& Yong Zhang) 333-368 (Springer International Publishing, 2016).

49 https://www.ce.tku.edu.tw/ icyeh/spcn/.

50 Ong, S. P. et al. Python Materials Genomics (pymatgen): A robust, open-source python library for materials analysis. Computational Materials Science 68, 314-319, doi:https://doi.org/10.1016/i.commatsci.2012.10.028 (2013). 


\section{Figure Captions}

Fig. 1 Schematic of the main concepts of the experiment. The blue region indicates that the physical properties of the binary and ternary spinel compounds are calculated by using the DFT calculations. The green area indicates that the non-stoichiometric compound to be calculated requires the creation of a stoichiometric compound first, thus the computational load is heavy. The red region indicates that the calculated physical properties of binary and ternary spinel compounds are used as machine learning input files, and then the predicted physical properties of the non-stoichiometric compound are obtained by using machine learning.

Fig. 2 Predicted lattice constants of the spinel-structured HEOx with different metal cations in the A and $\mathrm{B}$ sites.

Fig. 3 Predicted lattice constants of the spinel-structured HEOx with different proportions of metal cations in the $\mathrm{A}$ and $\mathrm{B}$ sites.

Fig. 4 Predicted formation energies of the spinel-structured HEOx with different metal cations in the $\mathrm{A}$ and $\mathrm{B}$ sites.

Fig. 5 Predicted formation energies of the spinel-structured HEOx with different proportions of metal cations in the $\mathrm{A}$ and $\mathrm{B}$ sites.

Fig. 6 (a) Lattice constants and (b) formation energies from DFT calculation (dark grey) and ML prediction (light grey). 
Figures

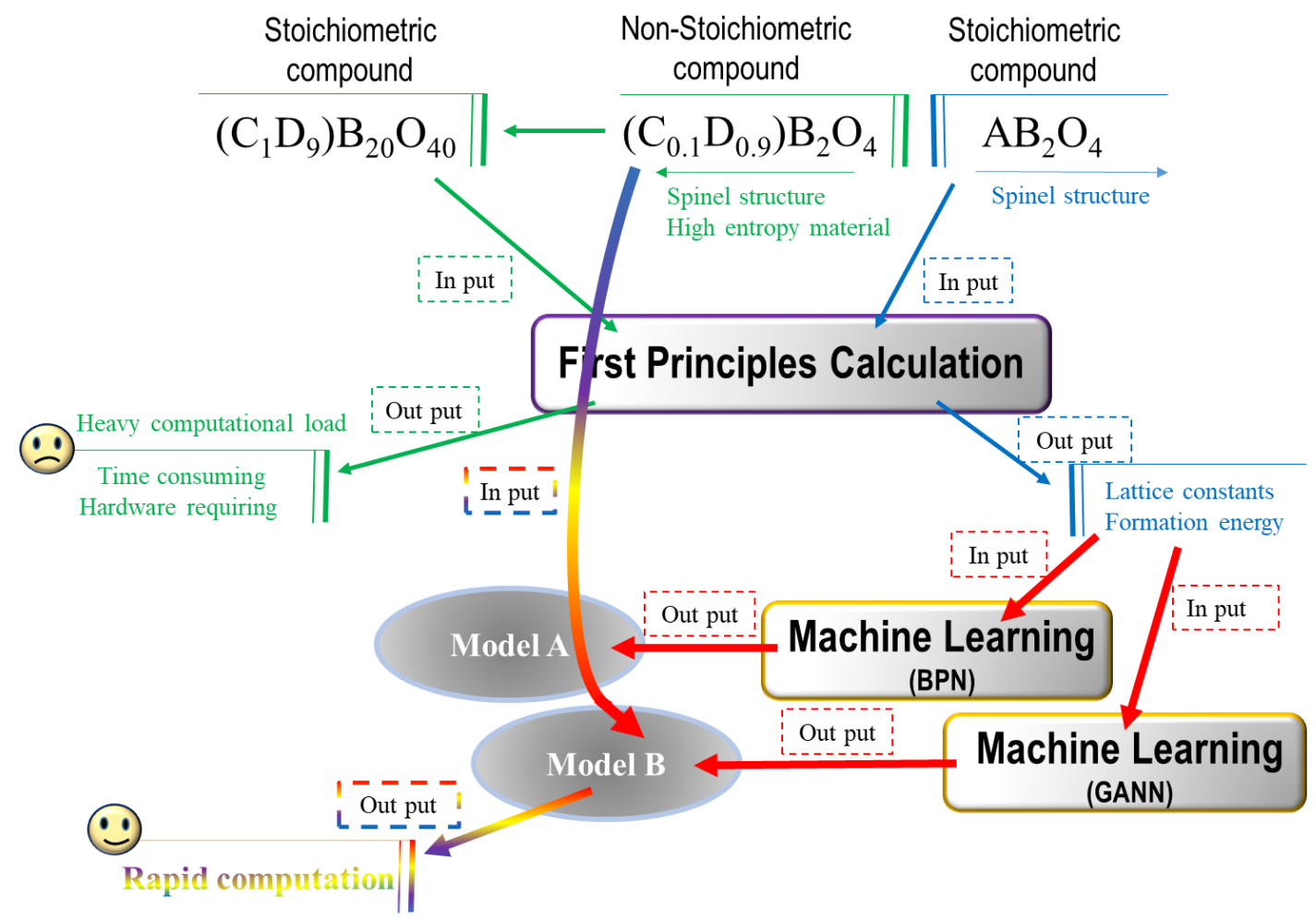

Fig. 1 Schematic of the main concepts of the experiment. The blue region indicates that the physical properties of the binary and ternary spinel compounds are calculated by using the DFT calculations. The green area indicates that the non-stoichiometric compound to be calculated requires the creation of a stoichiometric compound first, thus the computational load is heavy. The red region indicates that the calculated physical properties of binary and ternary spinel compounds are used as machine learning input files, and then the predicted physical properties of the non-stoichiometric compound are obtained by using machine learning. 


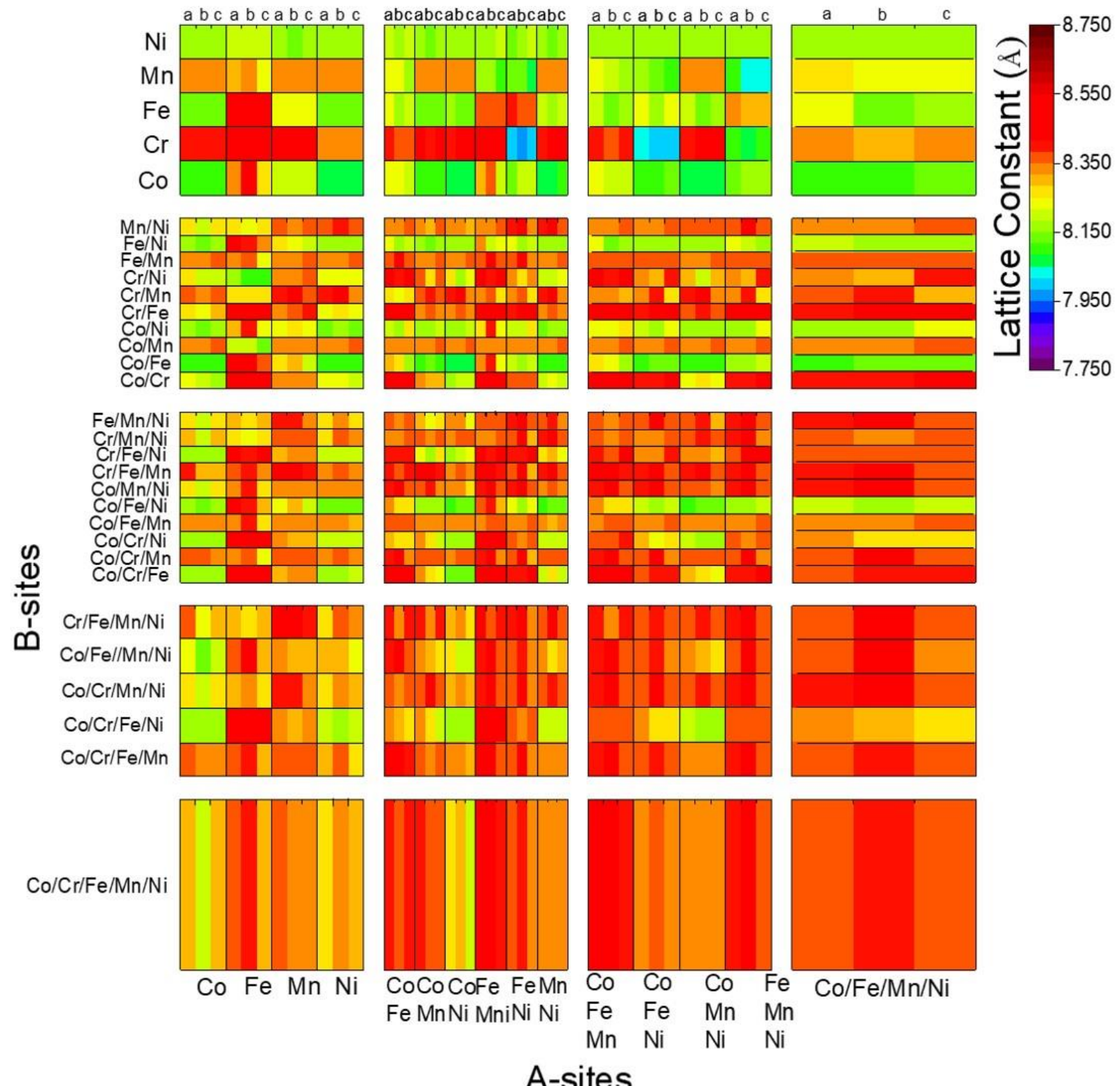

Fig. 2 Predicted lattice constants of the spinel-structured HEOx with different metal cations in the A and B sites. 

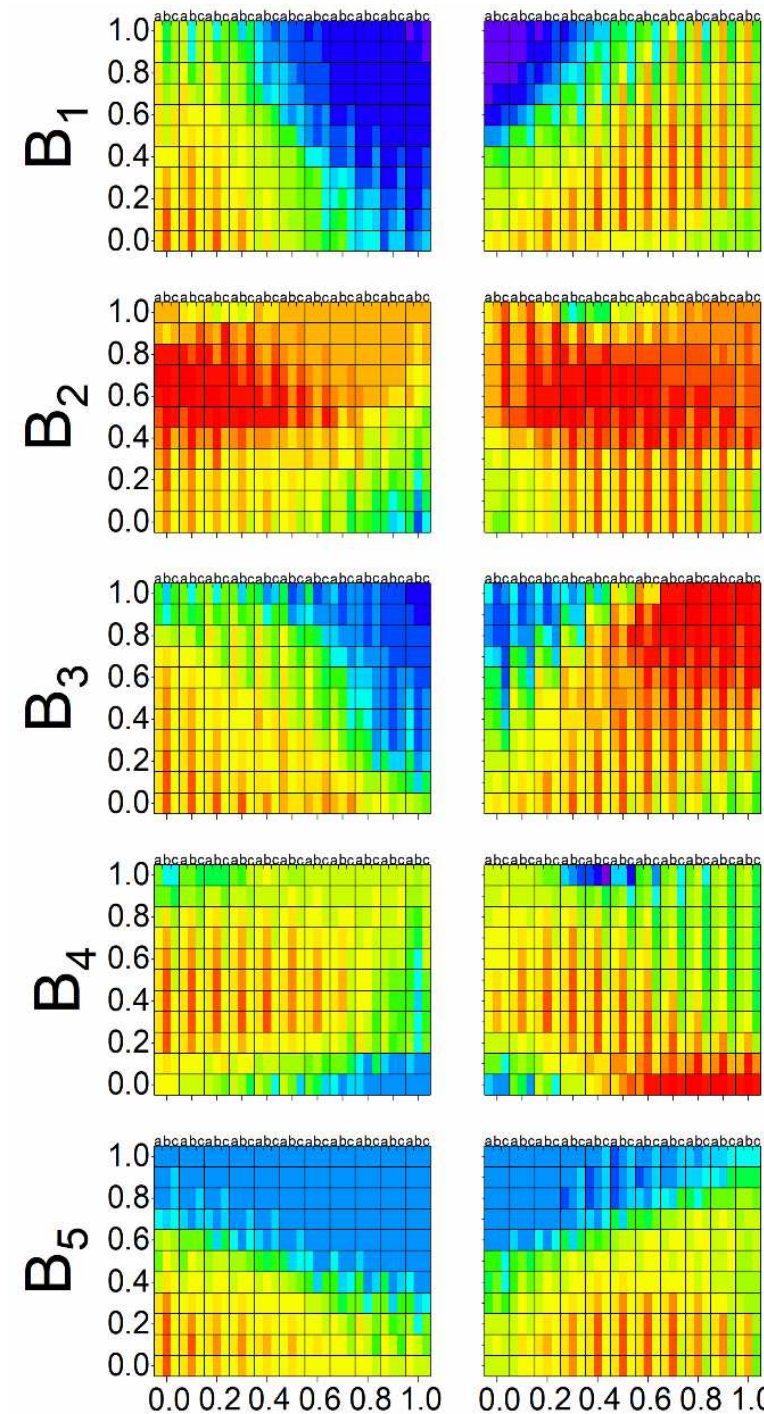

$\mathrm{A}_{1}$
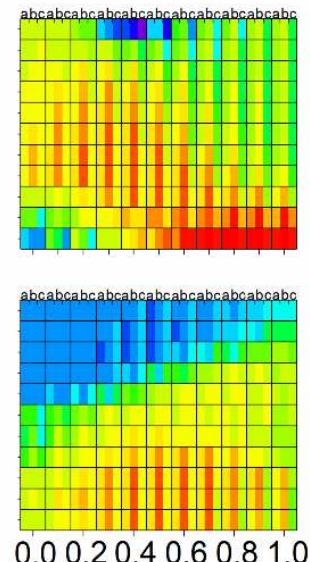

$\mathrm{A}_{2}$
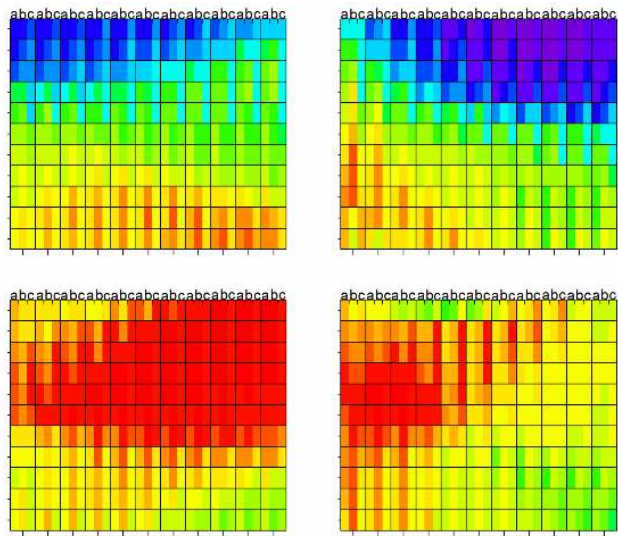

8.750
藏 8.600

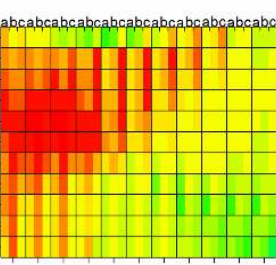

8.450
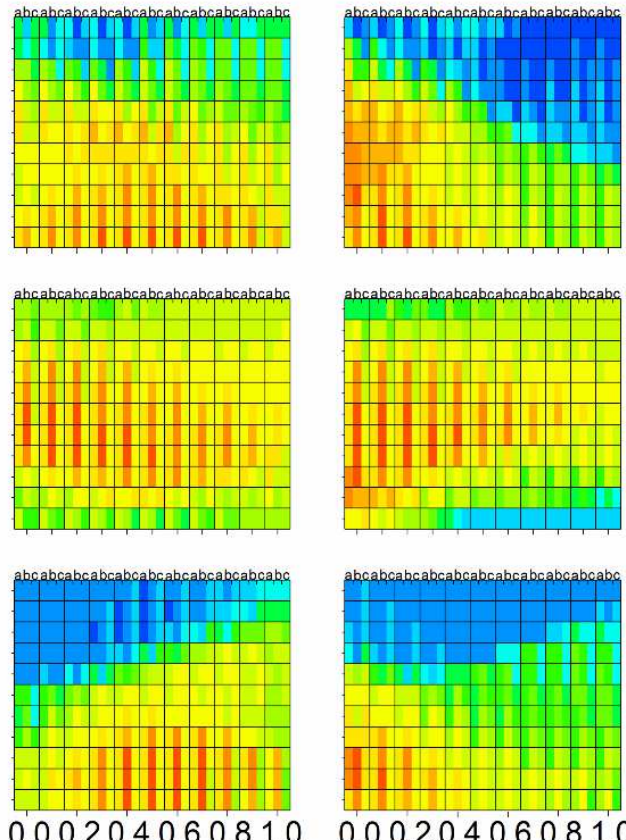

$\mathrm{A}_{3}$

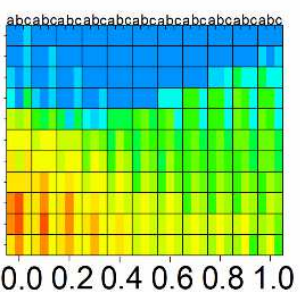

$\mathrm{A}_{4}$

Fig. 3 Predicted lattice constants of the spinel-structured HEOx with different proportions of metal cations in the A and B sites. 


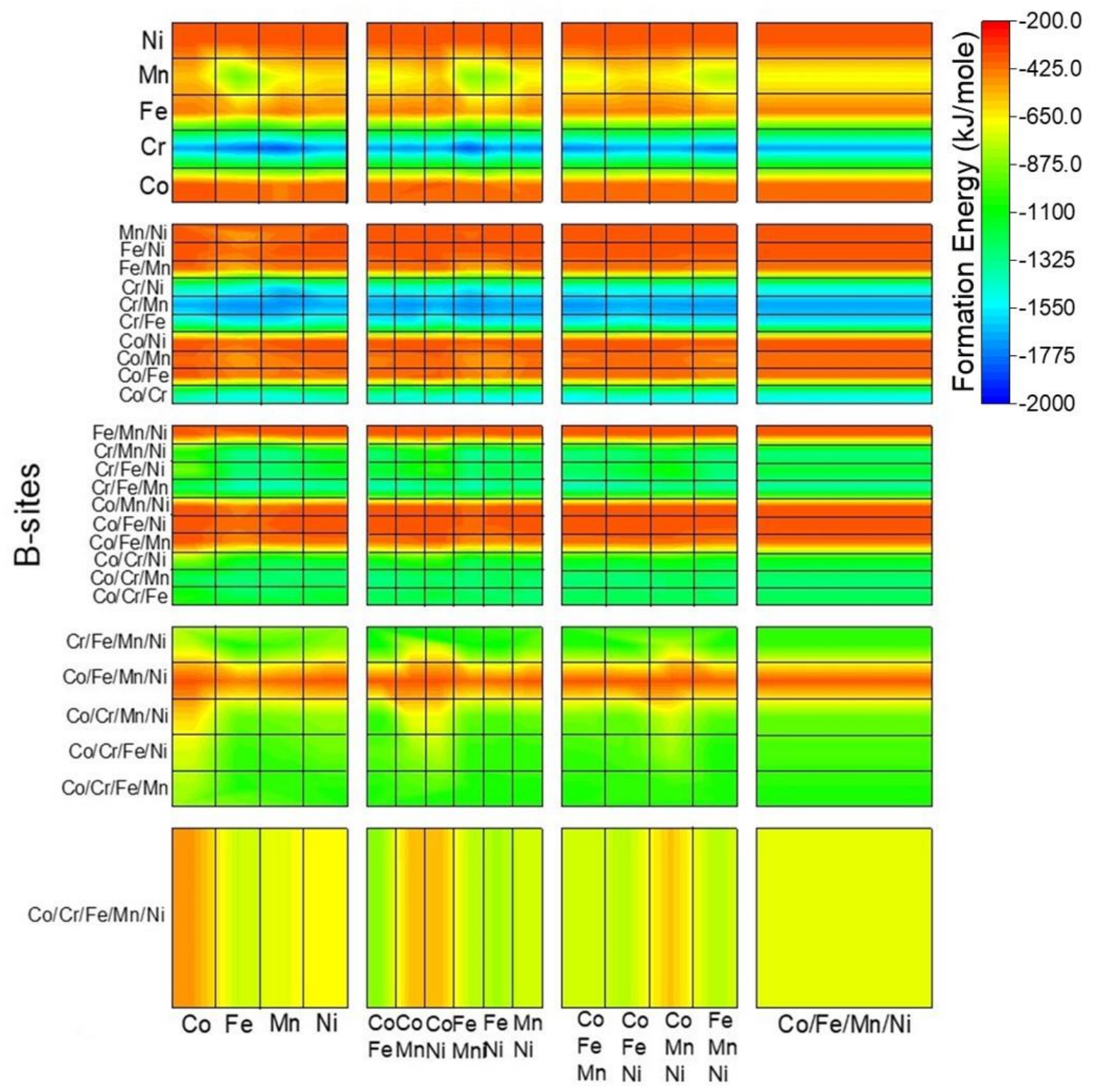

\section{A-sites}

Fig. 4 Predicted formation energies of the spinel-structured HEOx with different metal cations in the $\mathrm{A}$ and $\mathrm{B}$ sites. 


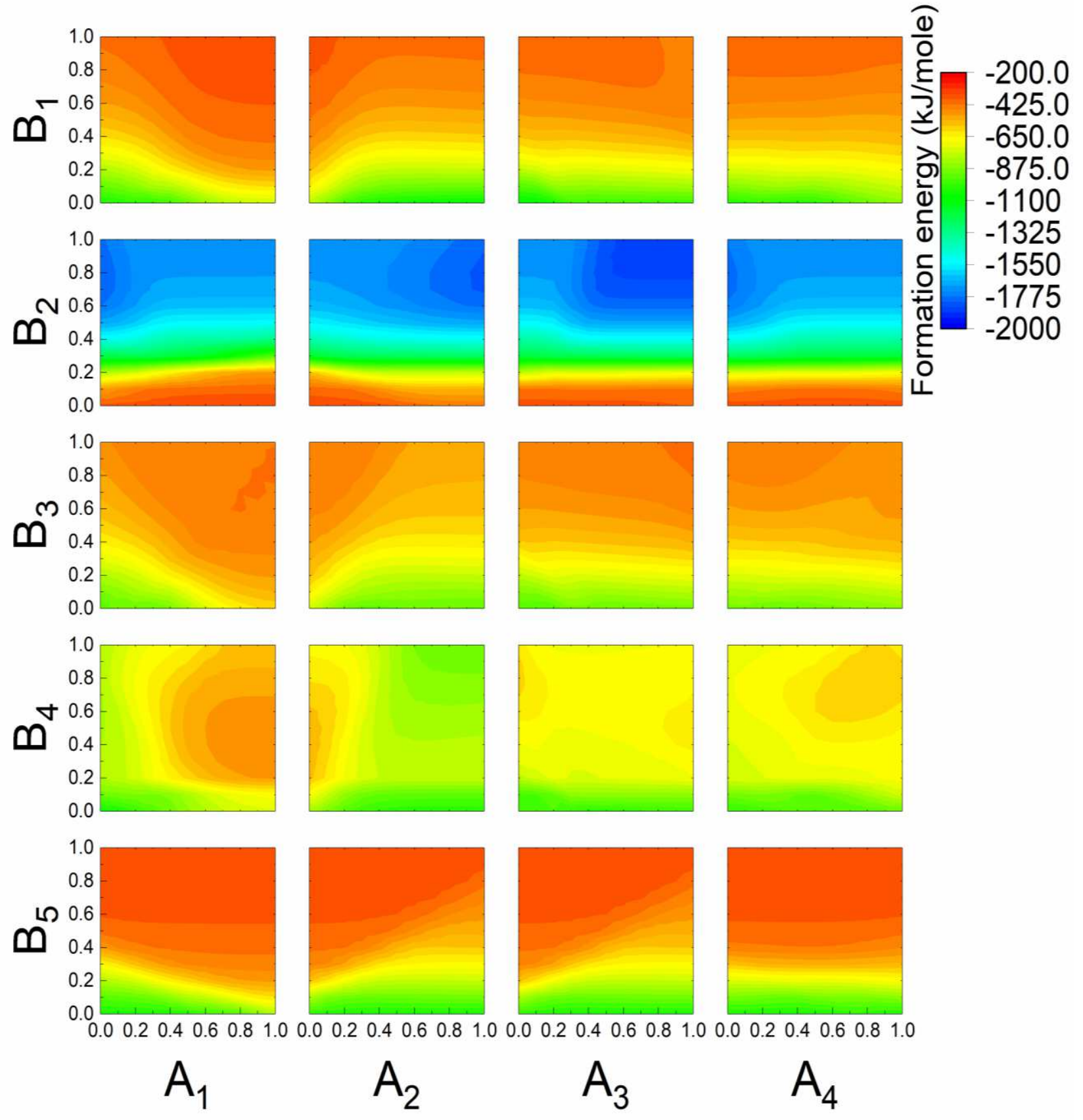

Fig. 5 Predicted formation energies of the spinel-structured HEOx with different proportions of metal cations in the A and B sites. 


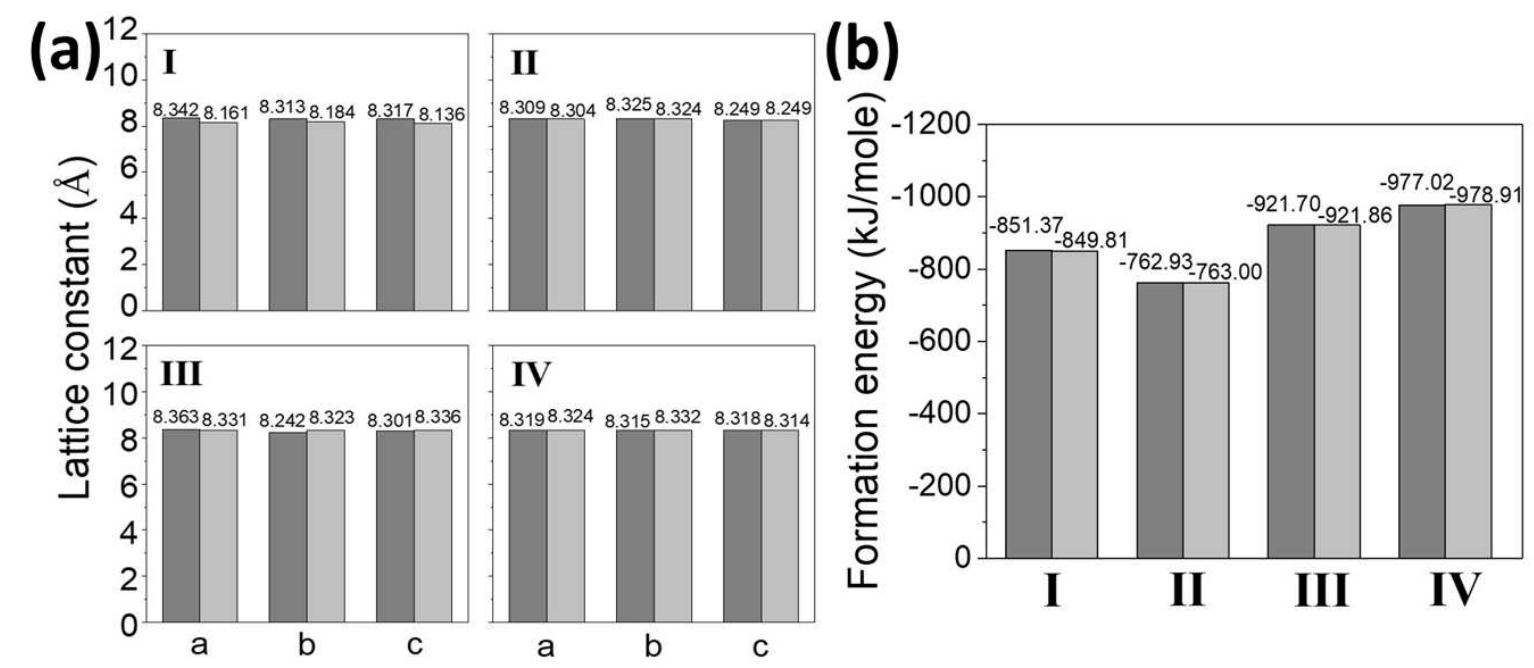

Fig. 6 (a) Lattice constants and (b) formation energies from DFT calculation (dark grey) and ML prediction (light grey). 


\section{Figures}

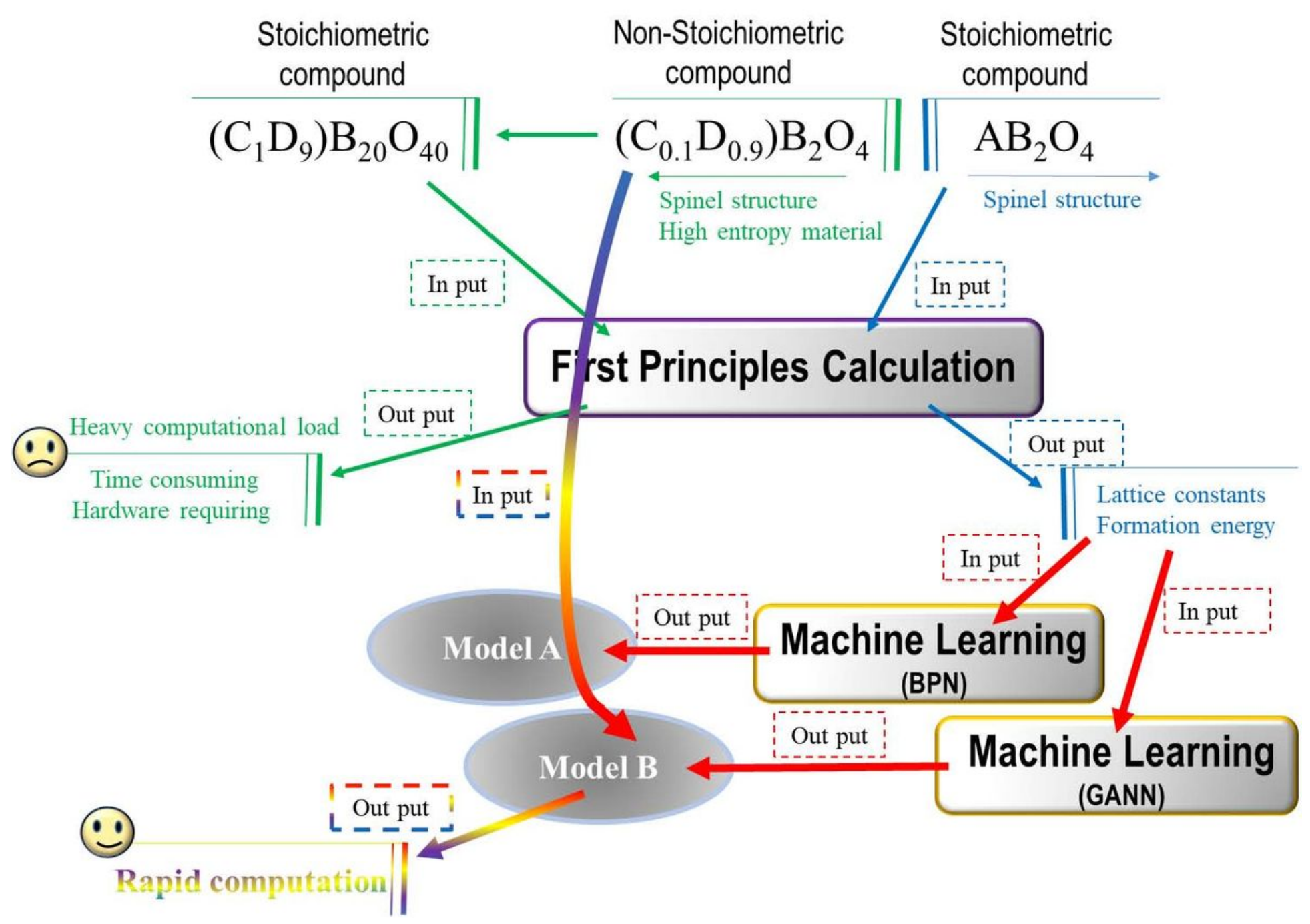

Figure 1

Schematic of the main concepts of the experiment. The blue region indicates that the physical properties of the binary and ternary spinel compounds are calculated by using the DFT calculations. The green area indicates that the non-stoichiometric compound to be calculated requires the creation of a stoichiometric compound first, thus the computational load is heavy. The red region indicates that the calculated physical properties of binary and ternary spinel compounds are used as machine learning input files, and then the predicted physical properties of the non-stoichiometric compound are obtained by using machine learning. 

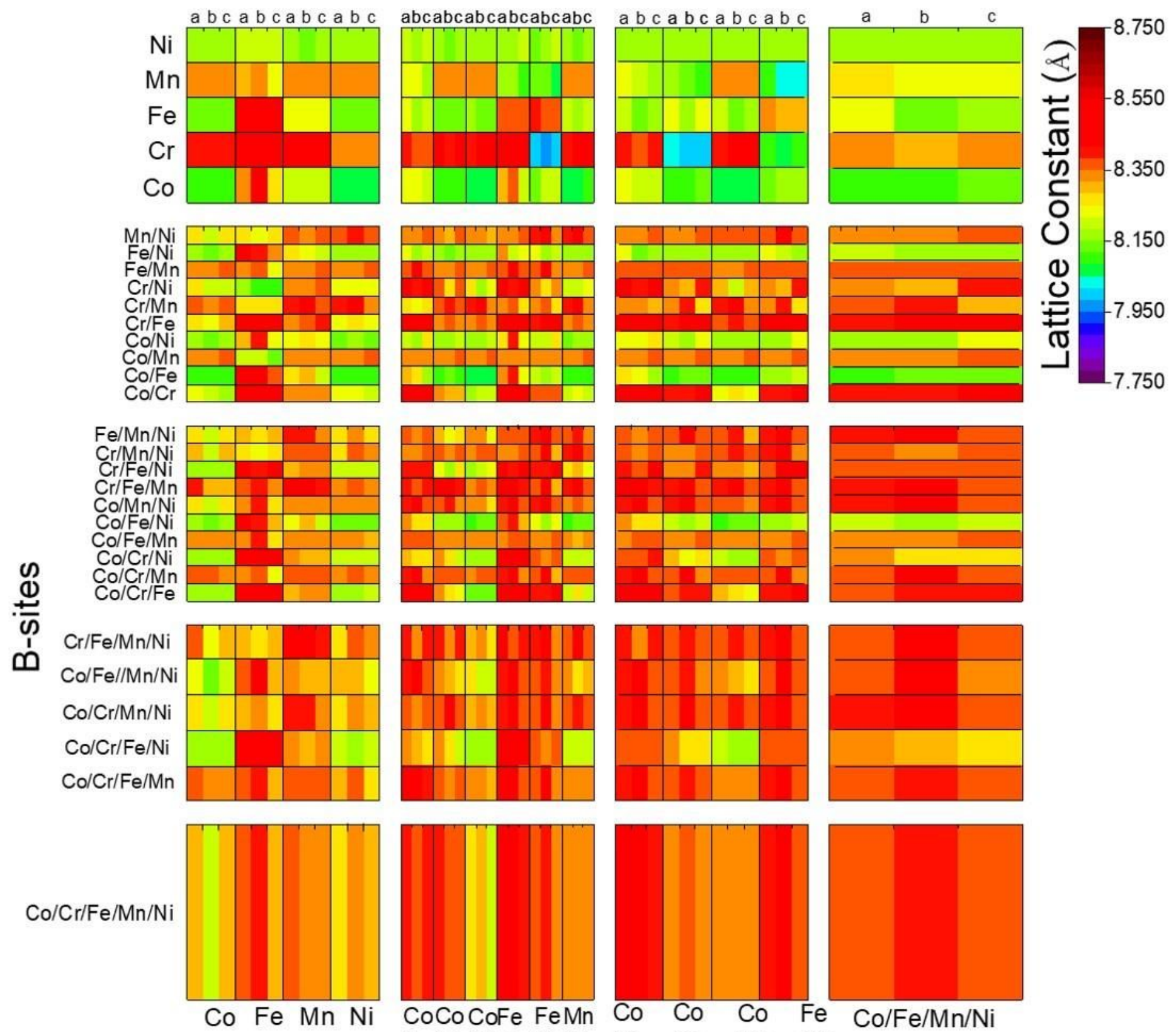

CoCoCoFe FeMn $\mathrm{Fe} \mathrm{MnNi} \mathrm{MniNi} \mathrm{Ni}$

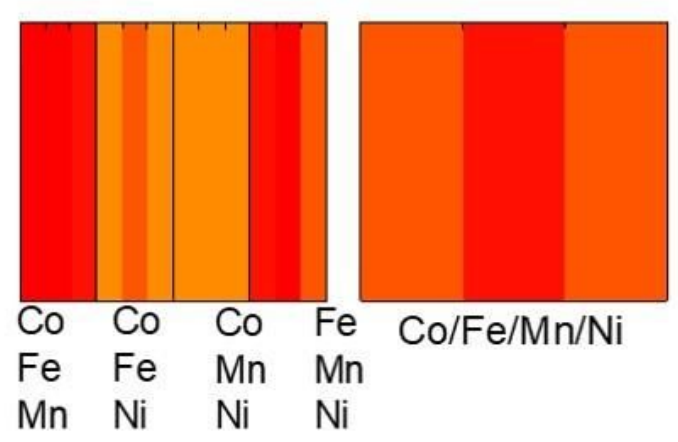

A-sites

\section{Figure 2}

Predicted lattice constants of the spinel-structured HEOx with different metal cations in the A and B sites. 

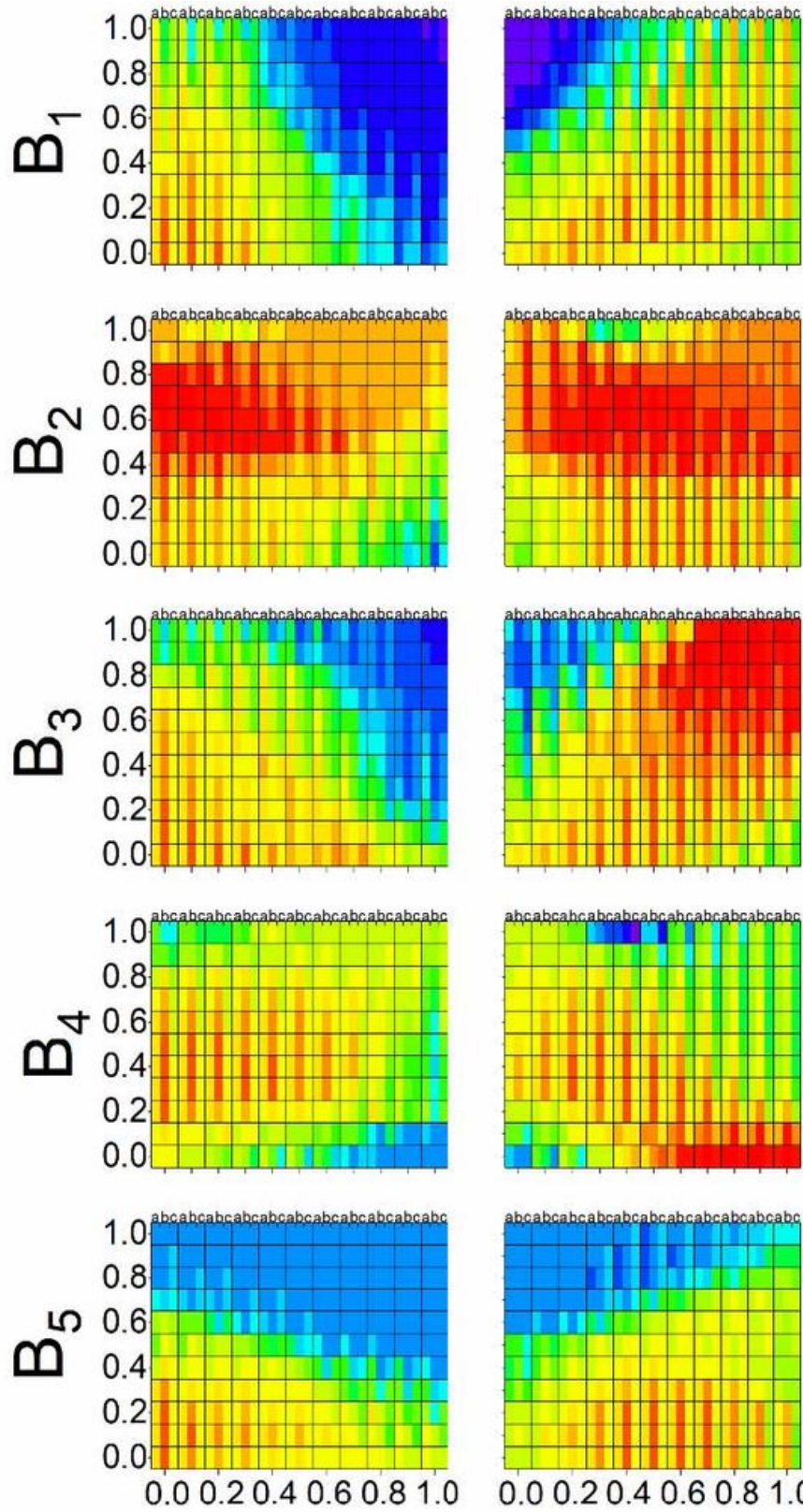

$\mathrm{A}_{1}$
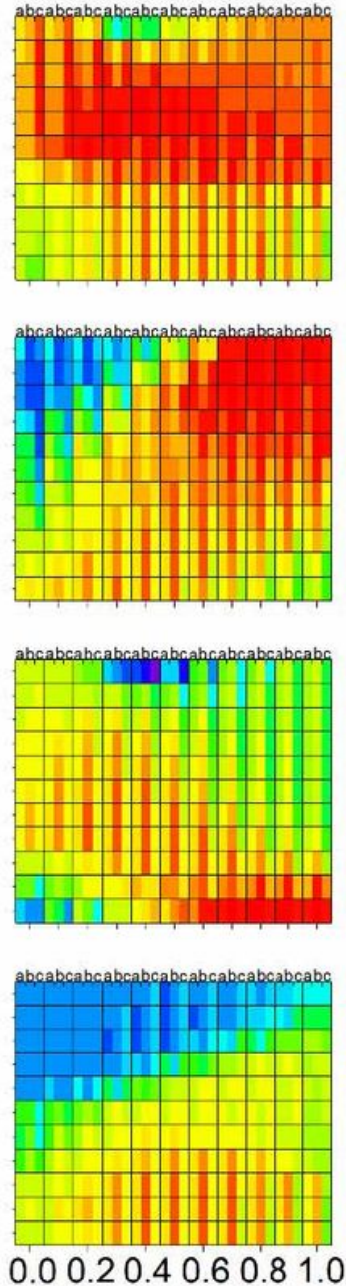

$\mathrm{A}_{2}$
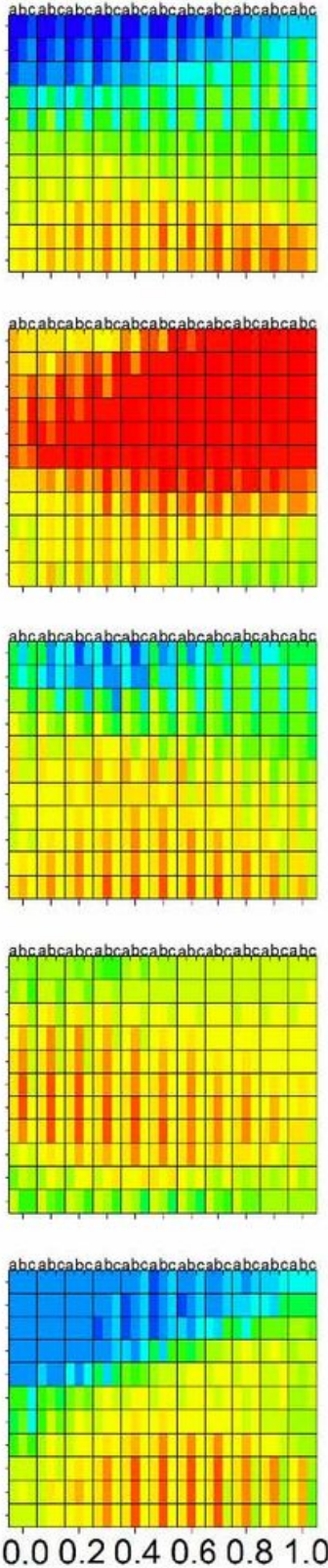

$\mathrm{A}_{3}$
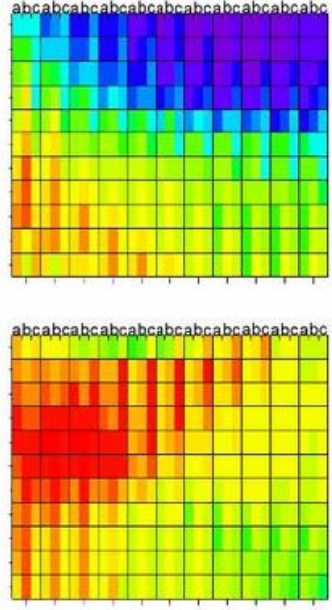

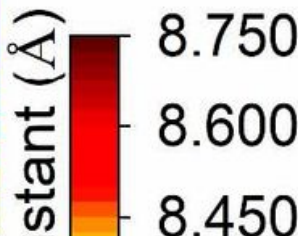
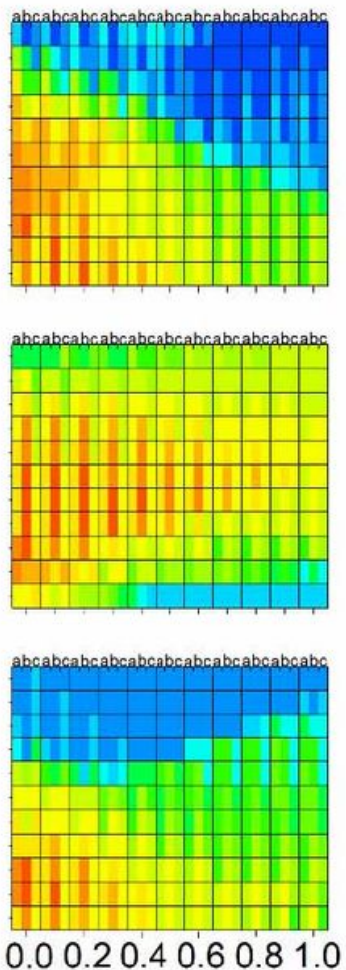

$\mathrm{A}_{4}$

Figure 3

Predicted lattice constants of the spinel-structured HEOx with different proportions of metal cations in the $A$ and $B$ sites. 


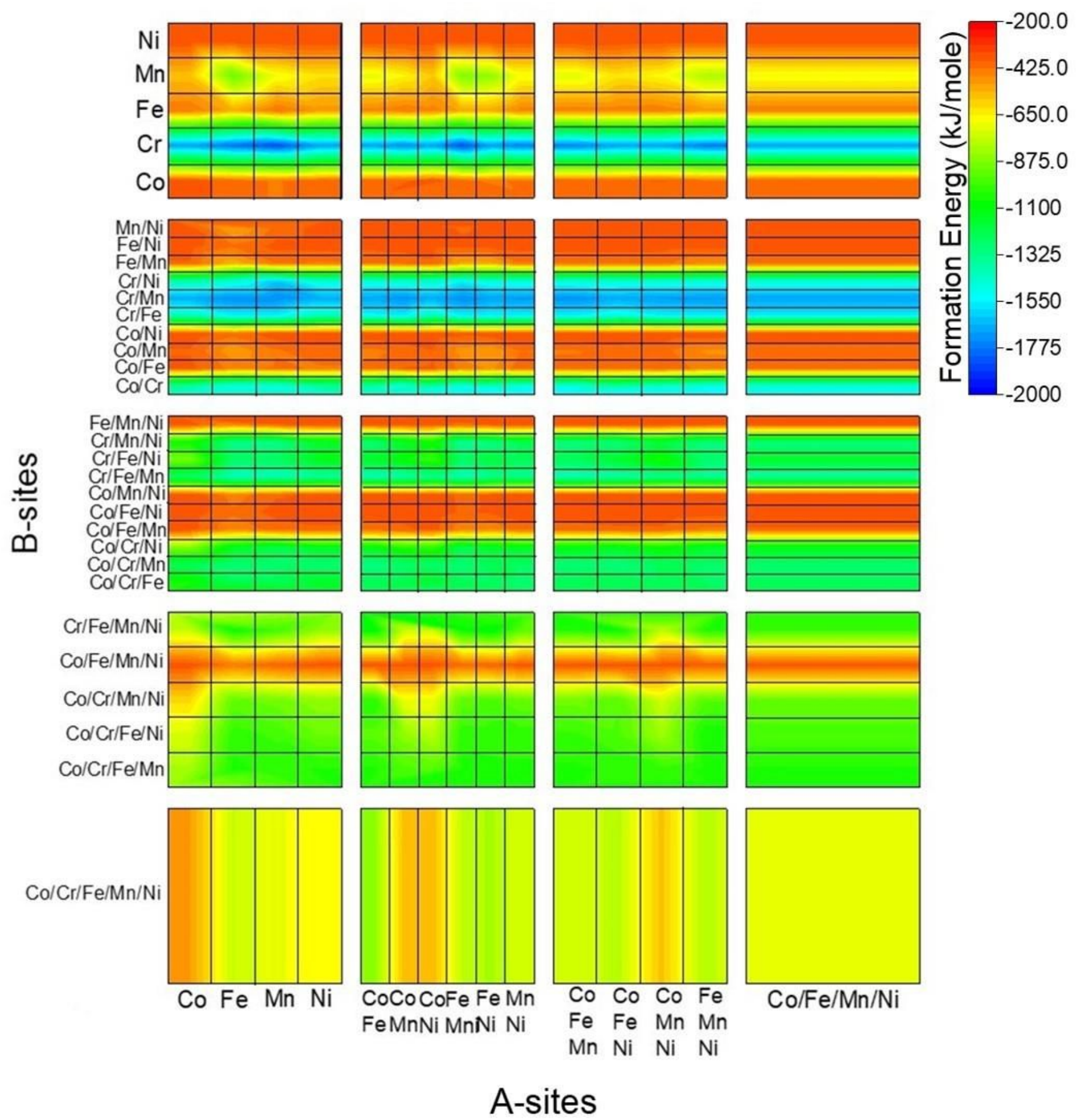

Figure 4

Predicted formation energies of the spinel-structured HEOx with different metal cations in the $A$ and $B$ sites. 

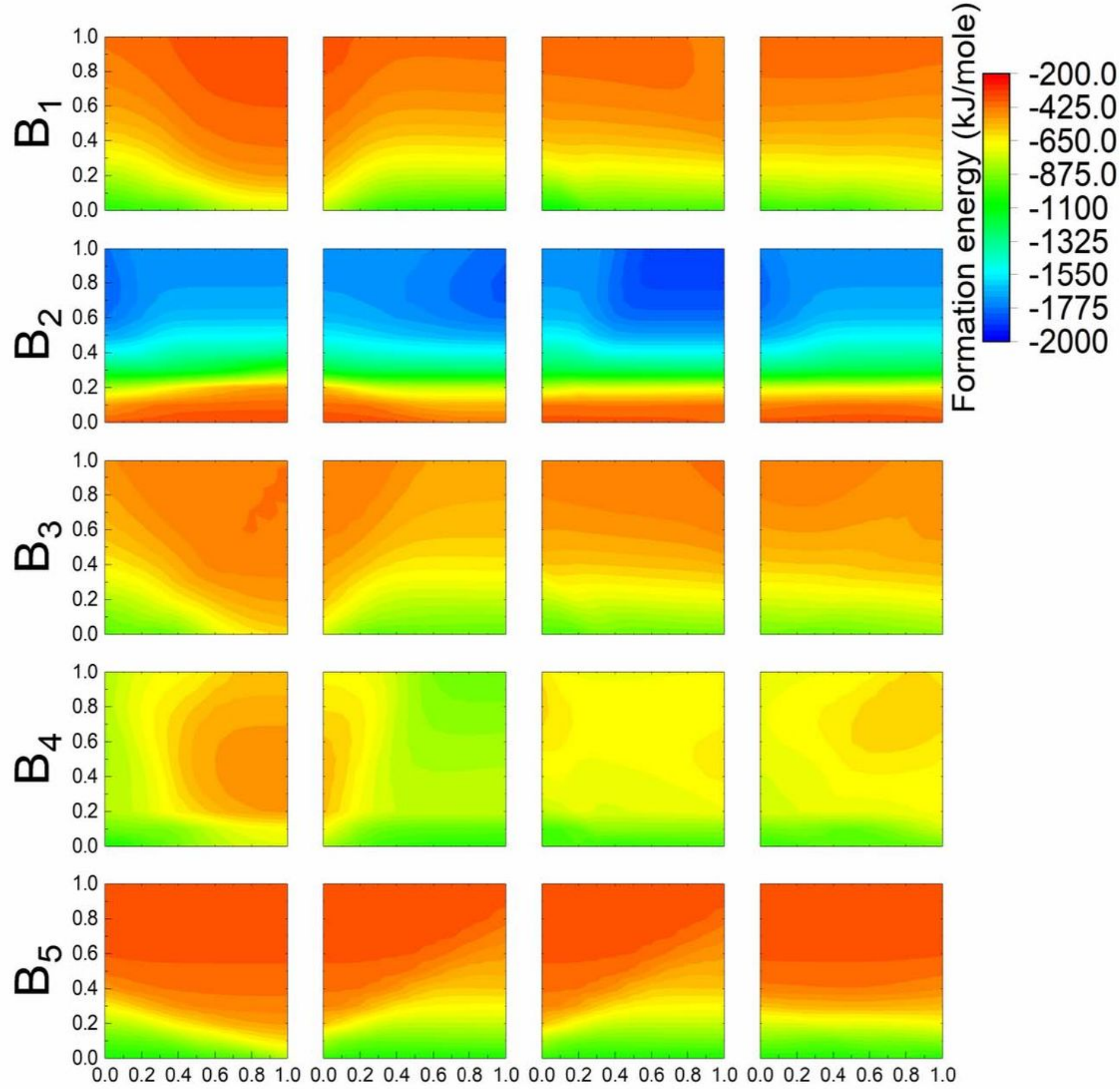

$\mathrm{A}_{1}$

$\mathrm{A}_{2}$

$\mathrm{A}_{3}$

$\mathrm{A}_{4}$

Figure 5

Predicted formation energies of the spinel-structured HEOx with different proportions of metal cations in the $A$ and $B$ sites. 


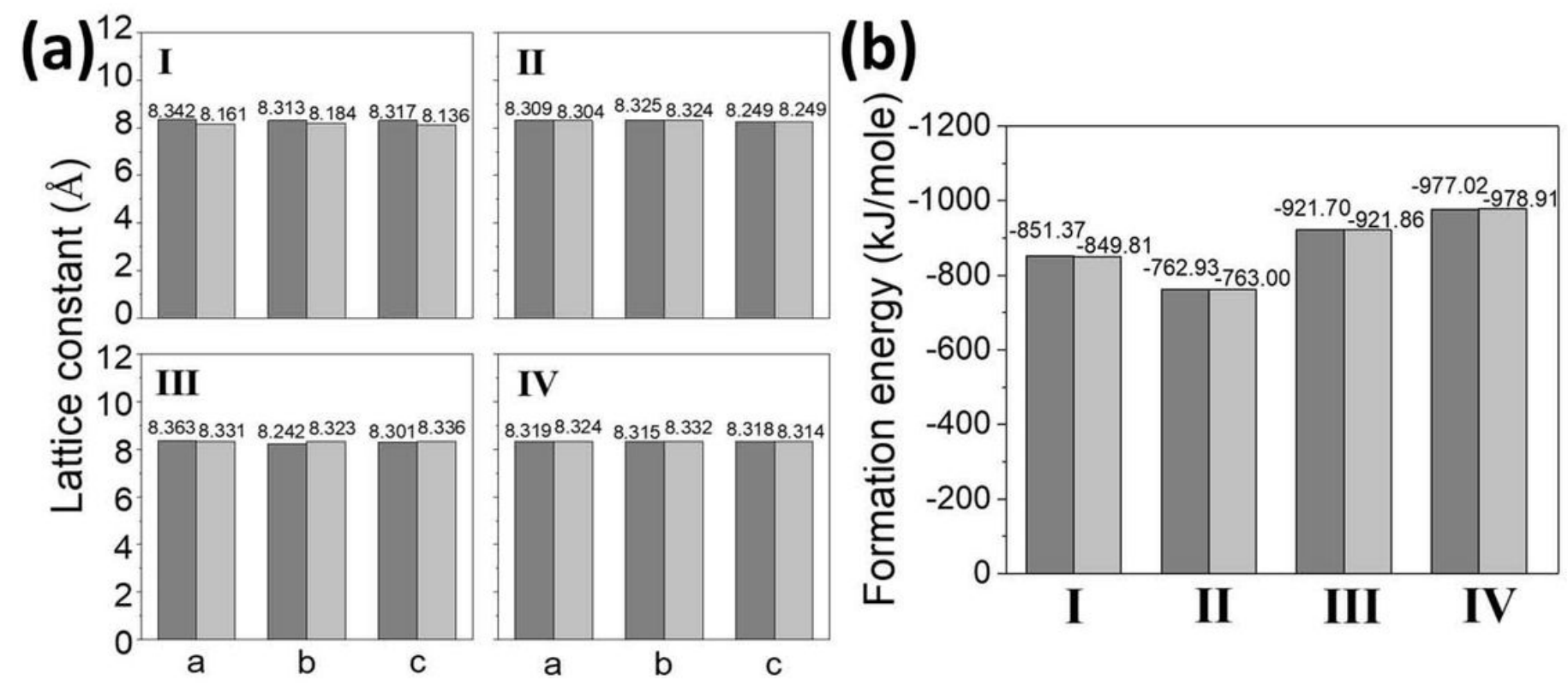

Figure 6

(a) Lattice constants and (b) formation energies from DFT calculation (dark grey) and ML prediction (light grey).

\section{Supplementary Files}

This is a list of supplementary files associated with this preprint. Click to download.

- SupportingInformation.pdf 